\title{
Correlative Light and Electron Microscopy (CLEM) on Biological Samples Using Immuno Electron Microscopy
}

\author{
C. ten Brink ${ }^{1}$, V. Oorschot ${ }^{1,2}$, J. Klumperman ${ }^{1}$ \\ ${ }^{1}$ Dept of Cell Biology, Cell Microscopy Core, University Medical Center Utrecht, \\ Heidelberglaan 100, Utrecht, The Netherlands. \\ ${ }^{2}$ Current address: Monash University, Melbourne, Australia
}

Correlative light and electron microscopy (CLEM) techniques integrate light microscopy (LM) and electron microscopy (EM) on a single sample. Application of CLEM on fixed samples often involves the use of fluorescent microscopy to define regions-of-interest that are subsequently traced back in the EM to provide subcellular context information (e.g. membrane organization, non-labeled surroundings. CLEM of live samples is very powerful since it infers dynamic information to static EM pictures. In addition, CLEM can be used to identify transient or rare events in live cells for high resolution analysis. By merging LM and EM an integrated set of information is obtained that cannot - or not easily - be achieved when using separate images of related events. However, imaging requirements are intrinsically different between LM and EM which is why creating conditions that are ideal for both modalities can be tedious and laborintensive. Our lab is specialized in the development of new probes and CLEM pipelines. We focus on methods that allow us to define the membrane compartments or membrane sub-domains that contain fluorescently-tagged proteins previously localized in fixed or living cells. We apply our technologies to study the cellular pathways and mechanisms that control the cell's digestive systems - i.e. the endo-lysosomal system - in health and disease conditions.

Our main CLEM technology is based on the use of immunogold labeling of ultrathin cryosections (Tokuyasu technique) [1]. Essential for CLEM of live cells is to prepare sections perpendicular to the plane of growth/filming. To this end, we developed a specialized 'flat embedding' protocol for Tokuyasu sections[2], which we have adapted to live cells[3]. Here we apply these techniques to perform CLEM.

An example of CLEM of live cells using Tokuyasu sections is shown in Figure 1. HeLa cells containing GFP-tagged LAMP1, a lysosomal membrane protein, were imaged alive and subsequently fixed, cryosectioned and labelled with antiGFP and protein A conjugated to $10 \mathrm{~nm}$ gold particles. Hence, membrane structures containing LAMP1-GFP were first followed in live cells, after which their ultrastructure was obtained by immunoEM. We currently use this technique to make a comprehensive overview of the dynamics of distinct endo-lysosomal intermediates. Figure 2 shows CLEM of fixed HeLa cells containing LAMP1-GFP. Ultrathin cryosections were prepared and labelled with antiGFP that was marked with both Alexa488conjugated secondary antibody as well as with protein A-10 nm gold [4]. CLEM allows us to select by LM cell profiles of interest in ultrathin sections after which detailed information is obtained by EM.

CLEM using immunoEM is a powerful method, but in order to increase the throughput of this methodology improvements in efficiency and accuracy are highly warranted. One restriction of current CLEM technologies is the limited resolution of LM since most set-ups use conventional LM. To circumvent these limitations we are currently adapting the CLEM approach shown in Figure 2 to super-resolution microscopy. 


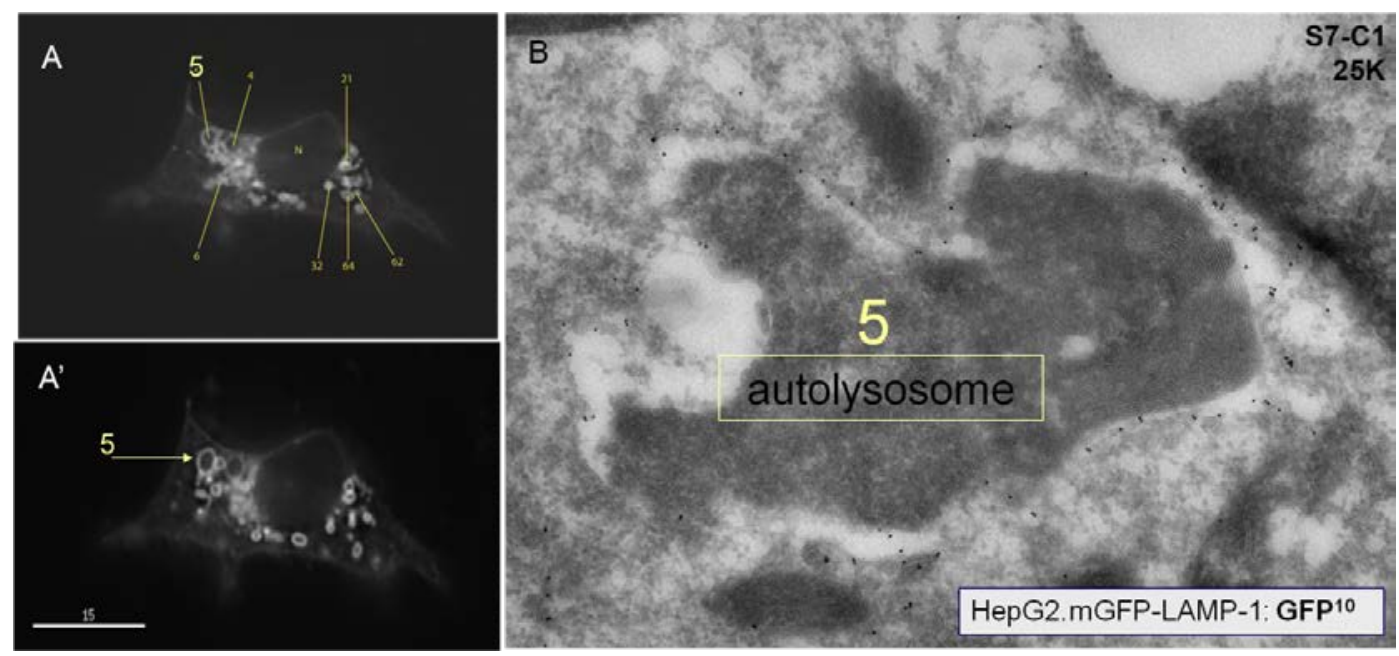

Figure 1. CLEM of live cells. A, A': Stills from movie of HepG2 cells transfected with mGFP.LAMP-1. Distinct LAMP-1 positive endo-lysosomal intermediates were followed over time by LM. B. EM picture of compartment 5 shown in A/A'. Cells were fixed in 2\% formaldehyde, prepared for ultrathin cyrosectioning and immunogold labeled (10 nm gold) for GFP. The high resolution morphology of compartment 5 is typical for autolysosomes.

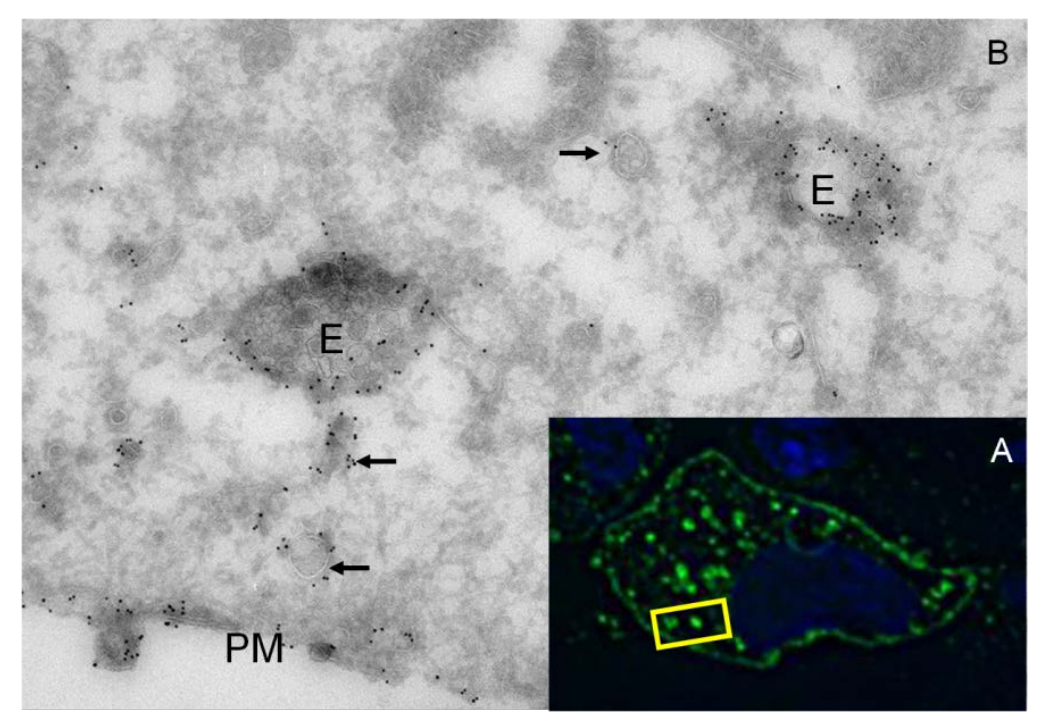

\section{References}

1. Slot, J.W. and H.J. Geuze, Cryosectioning and immunolabeling. Nature protocols, 2007. 2(10): p. 2480-2491.

2. Oorschot, V., et al., A novel flat-embedding method to prepare ultrathin cryosections from cultured cells in their in situ orientation. J Histochem Cytochem, 2002. 50(8): p. 1067-80.

3. van Rijnsoever, C., V. Oorschot, and J. Klumperman, Correlative light-electron microscopy (CLEM) combining live-cell imaging and immunolabeling of ultrathin cryosections. Nat Methods, 2008. 5(11): p. 973-80.

4. Cortese, K., et al., High data output method for 3-D correlative light-electron microscopy using ultrathin cryosections. Methods Mol Biol, 2013. 950: p. 417-37. 